\title{
Down-Regulation of miR-140 Induces EMT and Promotes Invasion by Targeting Slug in Esophageal Cancer
}

\author{
Weiling Lia Guojun Jiang ${ }^{\mathrm{b}}$ Jianping Zhou ${ }^{\mathrm{c}}$ Hongmin Wang ${ }^{\mathrm{b}}$ Zegang Gong ${ }^{\mathrm{b}}$ \\ Zheng Zhang ${ }^{d}$ Ke Mine Hongdi Zhu ${ }^{a}$ Yongfei Tan ${ }^{b}$ \\ aDepartment of Obstetrics and Gynecology, Affiliated Yixing Hospital of Jiangsu University, \\ bDepartment of Thoracic and Cardiovascular Surgery, Affiliated Yixing Hospital of Jiangsu University, \\ 'Department of Gastrointestinal Surgery, Affiliated Yixing Hospital of Jiangsu University, 'Department

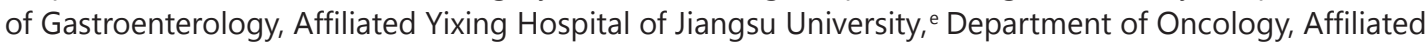 \\ Yixing Hospital of Jiangsu University, Yixing, People's Republic of China
}

\section{Key Words}

miR-140 • Slug • EMT • Invasion • Esophageal cancer

\begin{abstract}
Background/Aims: MicroRNAs (miRNAs) are reported to regulate cell invasion and functions by interfering with the translation of target mRNAs, but the role of miRNAs in esophageal cancer (EC) remains unclear. Methods: RT-PCR and Western blot were used to detect the expression of miRNAs and candidate genes in EC samples $(n=89)$. miR-140 mimics and inhibitor were tansfected in human TE-1 and Eca-109 cells. The transwell assay was used to examine the cell invasive ability. The regulation mechanism was confirmed by luciferase reporter assay. The markers of EMT were detected by using Western blot. Results: miR-140 expression was decreased in the EC tissues compared with the corresponding adjacent tumor tissues. Low expression of miR-140 promotes cell invasion by using transwell assay, while the effect of miR-140 high expression is reverse. Slug, a target gene of miR-140, was examined by luciferase assay and Western blot. Conclusions: miR-140 may regulate the cell invasion of EC via controlling Slug expression.
\end{abstract}

W. Li, G. Jiang and J. Zhou contributed equally to this work. 


\section{Introduction}

Esophageal cancer (EC) is a malignant tumor, which has become the sixth leading cause of death and the eighth most frequently diagnosed cancer [1]. Esophageal cancer contains two histological types, esophageal squamous cell carcinoma (ESCC) and esophageal adenocarcinoma (EAC) [2]. In China, a high incidence of ESCC exceeds 100 cases per 100,000 people per year. In contrast, esophageal adenocarcinoma (EAC) is the incidence of AC has increased steeply in Western countries [3]. Despite the rapid advancement in chemotherapy, radiation therapy and esophageal resection for EC, a 5-year overall survival rate has remained at about $14 \%$ due to the tumor metastasis [4]. Thus, elucidating the potential mechanism of tumor development would provide a new insight to understand the progression of EC.

A considerable number of studies have found that a large amount of aberrant expression level of genes participant in the development of EC. The interaction between these genes mostly exists in the transcription level and post-transcription level. microRNAs (miRNAs) were identified as an abundant class of small non-coding RNAs that played an important role in post-transcriptional regulation in different biological processes [5-7]. Here, functions of miR-140 related to cancers have been shown in recent studies. For example, miR-140 suppresses tumor growth and metastasis of non-small cell lung cancer by targeting insulinlike growth factor 1 receptor in NSCLC [8]. miR-140 is involved in the chemoresistance by reduced cell proliferation through $\mathrm{G}(1)$ and $\mathrm{G}(2)$ phase arrest mediated in part through the suppression of HDAC4 in human osteosarcoma and colon cancer cells [9].

Slug (Snail2) is one of transcription factors that play a central role in the activation of EMT [10]. Epithelial-to-mesenchymal transition (EMT) plays a pivotal role in invasion of various cancers by the transformation of polarized and adherent epithelial cells into motile and invasive mesenchymal cells $[11,12]$. This process of EMT is related to the downregulation of the epithelial marker E-cadherin and the upregulation of mesenchymal marker vimentin [13]. In addition, miRNAs function as crucial modulators for EMT. The miR-200 family and miR-205 have been identified as EMT-suppressive miRNAs directly targeting ZEB1, SIP1, or NCAM1, thereby reducing the aggressiveness of cancer cells $[14,15]$

In our study, we detected the expression of miR-140 in esophageal cancer and found that miR-140 was related to tumor metastasis and stage of esophageal cancer. Furthermore, we identified that miR-140 could regulate the invasion of EC cells by targeting Slug. Moreover, downregulation of miR-140 could induce EMT.

\section{Material and Methods}

\section{Clinical Samples}

A total of 89 patients with esophageal cancer had undergone routine surgery at Yixing Hospital of Jiangsu University from May 2008 and November 2012. Esophageal cancer samples and the corresponding adjacent esophageal tissues taken from the 89 patients were collected, immediately snap frozen in liquid nitrogen, and stored at $-80^{\circ} \mathrm{C}$ until RNA extraction. The tumors were classified according to World Health Organization classification. This study was approved by the Ethical Committee of Yixing Hospital of Jiangsu University, and every patient had written informed consent.

\section{Cell culture}

Eca-109 and TE-1 cell lines (ATCC) was employed for the present study and was cultured in RPMI1640 medium with $10 \%$ fetal bovine serum (Invitrogen, Carlsbad, CA) and penicillin (100 U/ml). Cells were cultured at $37^{\circ} \mathrm{C}$ with $5 \% \mathrm{CO}_{2}$.

Isolation of total RNA and Quantitative RT-PCR

Total RNA was extracted from EC tissues using TRIzol (Invitrogen, USA) and both miRNA and mRNA were reversely transcribed to cDNA. The expression levels of miRNAs were assessed by the TaqMan stem- 
loop RT-PCR method with a mirVana miRNA Detection Kit and gene-specific primers. U6 small nuclear RNA was used for normalization. Relative expression levels of E-cadherin, N-cadherin, Vimentin and Slug mRNA were examined by SYBR Green real-time PCR (RT-PCR) and normalized to GAPDH. RT-PCR was performed by using the ABI 7500 Fast Real-Time PCR system (ABI, CA, USA).

\section{Cell-Proliferation Assay (Cell-Counting Kit-8)}

Cells were seeded into 96 -well plates $\left(6.0 \times 10^{3}\right.$ cells per well). Cell viability was assessed by cellcounting kit-8 assay (Beyotime Institute of Biotechnology, Shanghai, China). The absorbance of each well was read on a spectrophotometer (Thermo) at $450 \mathrm{~nm}$. Three independent experiments were performed in quintuplicate.

\section{Transwell assay}

Cell motility was measured using an 8- $\mu \mathrm{m}$-pore polycarbonate membrane Boyden chamber insert in a Transwell apparatus (Millipore, MA, USA). The transfected cells were treated with trypsin/EDTA solution, washed once with serum-containing RPMI- 1640 medium. A total of $1 \times 10^{5}$ cells in $0.2 \mathrm{ml}$ serum-free RPMI1640 medium were seeded on a Transwell apparatus. RPMI-1640 containing 10\% FBS (600 $\mu$ l) was added to the lower chamber. An invasion assay was conducted following the same procedure, with the exception that the filters of the transwell chambers were coated with $45 \mu \mathrm{g}$ Matrigel (BD Biosciences; San Jose, CA, USA). Following incubation of the cells for $24 \mathrm{~h}$ at $37^{\circ} \mathrm{C}$ in a $5 \% \mathrm{CO}_{2}$ incubator, cells on the top surface of the insert were removed by wiping with a cotton swab. Cells that invaded to the bottom surface of the insert were fixed in the $100 \%$ precooling methanol for $10 \mathrm{~min}$, stained in $0.5 \%$ crystal violet for $30 \mathrm{~min}$, then rinsed in PBS and subjected to microscopic inspection. The values for invasion were obtained by counting three fields per membrane and represented the average of three independent experiments.

\section{Western blot}

For western-blotting, total proteins were extracted from tissues or cultured cells using RIPA buffer containing PMSF (Roche, Basel, Switzerland). Total proteins were prepared from the established cells, quantities using a protein assay (BCA method, Beyotime, China). Proteins were fractionated by sodium dodecyl sulfate polyacrylamide gel electrophoresis (SDS-PAGE) transferred to polyvinylidene fluoride (PVDF) membrane, blocked in 5\% dry milk at room temperature for 1 hour and immunostained with antibodies at $4^{\circ} \mathrm{C}$ overnight using anti-Slug, anti-E-cadherin, anti-N-cadherin, anti-Vimentin (1:500, santa, USA) and anti-GAPDH (1:5000, kangcheng, China). All results were visualized through a chemiluminescent detection system (Pierce ECL Substrate Western blot detection system, Thermo, Pittsburgh, PA) and then exposed in Molecular Imager ChemiDoc XRS System (Bio-Rad, Hercules, CA). The integrated density of the band was quantified by Image software.

\section{Transient transfection}

The cells were seeded into 6-well plates and transfected with $50 \mathrm{nM}$ siRNA/Slug and siRNA/control (GenePharma, Shanghai, China) using Lipofectamine 2000 (Invitrogen, USA) according to the instructions provided by the manufacturer. Oligonucleotides hsa-miR-140 mimics (miR-140), NC (miR-control), hsamiR-140 inhibitor (anti-miR-140), inhibitor NC (anti-miR-control) were purchased from GenePharma (Shanghai, PR China). The transfection was conducted by using Lipofectamine 2000 Reagent (Invitrogen, Carlsbad, CA, USA) at a final concentration of $100 \mathrm{nM}$.

\section{Plasmid construction and cell transduction}

3'-UTR sequence of Slug which was predicted to interact with miR-140 or a mutant sequence with the predicted target sites was inserted into the KpnI and SacI sites of pGL3 promoter vector (Genscript, Nanjing, China). They were named pGL3-Slug and pGL3-Slug-mut. The cells were plated onto 6-well plates and were transfected with $100 \mathrm{ng}$ of pGL3-Slug or pGL3-Slug-mut, and miR-140 mimics (50nM) by using Lipofectamine 2000 (Invitrogen Corp, CA, USA). Slug gene was synthesized (purchased from Genscript, Piscataway, NJ) with restrictive digestion using Mlu I and subcloned pLV-GFP plasmid, and named pLV-GFPSlug. Recombinant lentivirus was generated from 293T cells using calcium phosphate precipitation. Cell lines were transfected with lentivirus using polybrene $(8 \mathrm{ug} / \mathrm{ml})$. 


\begin{tabular}{|c|c|c|}
\hline \multirow{2}{*}{$\begin{array}{l}\text { Cellular Physiolosy } \\
\text { and Biochemistry }\end{array}$} & \multirow{2}{*}{\multicolumn{2}{|c|}{$\begin{array}{l}\text { Cell Physiol Biochem 2014;34:1466-1476 } \\
\begin{array}{l}\text { DOI: 10.1159/000366351 } \\
\text { O 2014 S. Karger AG, Basel }\end{array}\end{array}$}} \\
\hline & & $\begin{array}{l}\text { O } 2014 \text { S. Karger AG, Basel } \\
\text { www.karger.com/cpb }\end{array}$ \\
\hline
\end{tabular}

Table 1. Expression levels of miR-140 in EC and corresponding adjacent tissues. ${ }^{\mathrm{a}}$ Median $=0.3015, *$ indicates $\mathrm{P}<0.05$

\begin{tabular}{|c|c|c|c|c|}
\hline Characteristics & All Patients & $\begin{array}{c}\text { miR-140 low expression } \\
\left(\leq \text { Median }^{\mathrm{a}}\right)\end{array}$ & $\begin{array}{c}\text { miR-140 high expression } \\
\left(>\text { Mediana }^{2}\right)\end{array}$ & p value \\
\hline $\mathrm{n}$ & 89 & 45 & 44 & \\
\hline Age(yr) & & & & 0.927 \\
\hline$<64$ & 38 & 19 & 19 & \\
\hline$\geq 64$ & 51 & 26 & 25 & \\
\hline Sex & & & & 0.741 \\
\hline Male & 40 & 21 & 19 & \\
\hline Female & 49 & 24 & 25 & \\
\hline Histology & & & & 0.762 \\
\hline Adenocarcinoma & 35 & 17 & 18 & \\
\hline Suqamous cell carcinoma & 54 & 28 & 26 & \\
\hline Tumor depth & & & & 0.167 \\
\hline Tis,T1 & 36 & 15 & 21 & \\
\hline $\mathrm{T} 2, \mathrm{~T} 3, \mathrm{~T} 4$ & 53 & 30 & 23 & \\
\hline Stage & & & & $0.007^{*}$ \\
\hline $0, \mathrm{I}$ & 34 & 11 & 23 & \\
\hline II ,III,IV & 55 & 34 & 21 & \\
\hline Metastasis & & & & $0.000^{*}$ \\
\hline No & 54 & 17 & 37 & \\
\hline Yes & 35 & 28 & 7 & \\
\hline
\end{tabular}

Luciferase activities analysis

48 hours after transfection, luciferase actions were measured in Victor 1420 Multilabel Counter (Wallac, Finland) using Luciferase Assay System (Promega, USA) according to the manufacturer's protocol.

\section{Statistical methods}

The method of 2- $\Delta$ Ct was used to analyze the results of RT-PCR in all the experiments performed in this study. Statistical analysis was performed using STATA 9.2, and presented with Graph PAD prism software. Experimental data of tissue samples are presented as box plot of the median and range of log-transformed relative expression level which was analyzed by Wilcoxon rank-sum (Mann-Whitney) test. While the results obtained from experiment in vitro assays are presented as mean \pm SE from three separate experiments in triplicates per experiment, and the data was analyzed by double-sided Student's t-test. Results were considered statistically significant at $\mathrm{P}<0.05$.

\section{Results}

miR-140 is decreased in human esophageal cancer tissues

First, we analyzed the expression levels of miR-140 in both ESCC tissues and adjacent tissues by qRT-PCR. Significantly, we found that miR-140 expression was decreased in ESCC tissues (n=89, Fig. 1A). All EC were divided into miR-140 low-expression group $(n=45)$ and high-expression group ( $\mathrm{n}=44)$, median ( ${ }^{\mathrm{a}}$ median $\left.=0.3015\right)$ was used as cut off. The correlation between miR-140 expression and clinicopathological characteristics is exhibited (Table 1). No positive correlation with gender, age, histological differentiation, however, there was significantly correlation with tumor stage $(\mathrm{p}=0.007)$ and metastasis $(\mathrm{p}<0.000)$. The aberrant expression level of miR-140 suggested that miR-140 may play an important role the pathogenesis of EC (Table 1). Furthermore, we also detected that the mRNA levels of E-cadherin, N-cadherin, Vimentin and Slug in both ESCC tissues and adjacent tissues by using qRT-PCR. The mRNA level of E-cadherin was reduced (Fig. 1B) while N-cadherin (Fig. 1C) and Vimentin (Fig. 1D) were increased in ESCC tissues. Therefore, based on this expression pattern, we chose the Eca-109 and TE-1 esophageal cancer cell lines to verify the effect of miR-140.

Aberrant expression level of miR-140 affects EC cell invasion but had no effect on cell proliferation in vitro

Eca-109 and TE-1 cell lines were transfected with miR-140 mimics, NC, miR-140 inhibitor and inhibitor NC respectively. The transfection efficiency was validated by qRT-PCR 
Fig. 1. $\operatorname{miR}-140$ is down-regulated in EC patients. A: The expression levels of miR-140 in human EC tissues and corresponding adjacent tissues relative to U6 were determined by qRT-PCR. (n=89, $\mathrm{p}<0.0001$ ). B, C and D: The mRNA level of $E$ cadherin, N-cadherin and Vimentin were determined by qRT-PCR. E: miR-140 expression level in cell lines transfected with miR140 mimics, miR-140 inhibitor, control for miR-mimics (NC) and control for miR-140 inhibitor (inhibitor NC). The result was validated by real-time PCR. Data are represented as mean \pm SEM. * indicates $\mathrm{P}<0.05$.
A

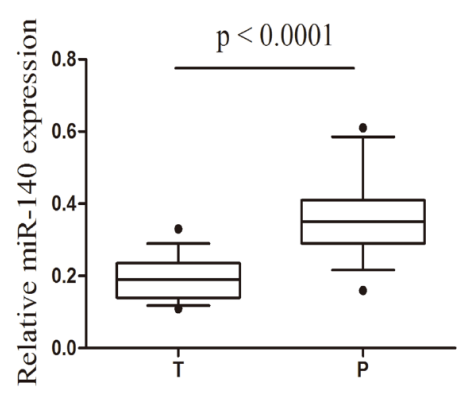

$\mathrm{C}$

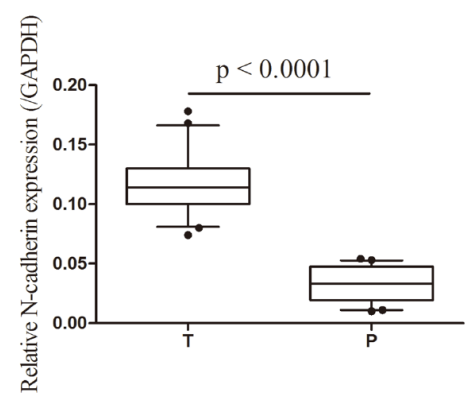

B

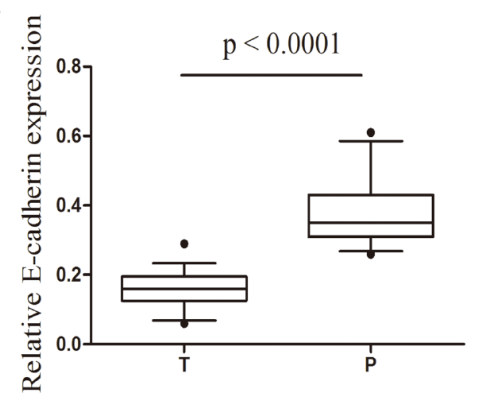

$\mathrm{D}$

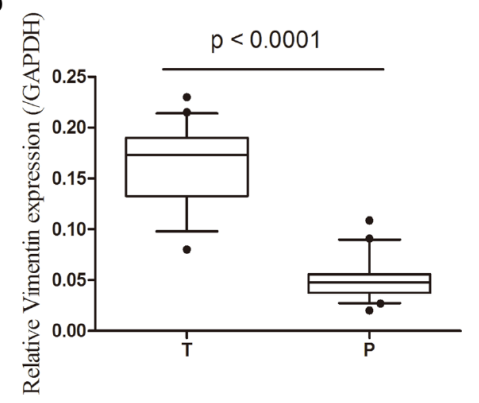

$\mathrm{E}$

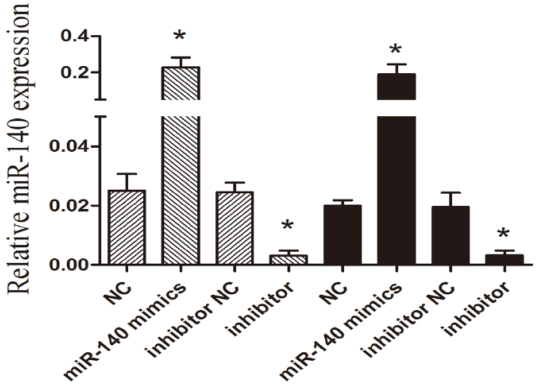

(Fig. 1E). Matrigel invasion assay showed that overexpression of miR-140 attenuated Eca109 and TE-1 cell lines invasion, whereas the suppressed miR-140 promoted the invasion ability of EC cell lines (Fig. 2A). We also used CCK8 assay to detect the effect of miR-140 on cell proliferation. To investigate whether miR-140 could regulate EC cell lines proliferation in vitro, the effect of miR-140 on cell proliferation was detected by using CCK8 assay. The cell-proliferation assay test showed distinct differences on proliferation after manipulation of miR-140 in both TE-1 and Eca-109 cells at 12-hour, 24-hour, and 48-hour time points. The assay showed that miR-140 overexpression or downregulation did not regulate cell proliferation (Fig. 2B). The results suggested that miR-140 may play an important role in inhibiting EC metastasis.

\section{miR-140 could regulate EMT in vitro}

Epithelial-to-mesenchymal transition (EMT) has been identified to contribute to the cell invasion of different cancers by the transformation of polarized and adherent epithelial cells into motile and invasive mesenchymal cells. To determine whether miR-140 could regulate the molecular changes of EMT in EC cells, the expression of mesenchymal markers, including $\mathrm{N}$-cadherin and Vimentin and the epithelial marker, E-cadherin, was examined in the cells by using western blot. Thus, Eca-109 cells were transfected with NC, miR-140 mimics, inhibitor NC and miR-140 inhibitor. In the protein expression level, upregulated miR-140 expression resulted in evaluated E-cadherin expression and decreased N-cadherin, Vimentin and Slug expression. Meanwhile, suppressed miR-140 expression resulted in decreased E-cadherin expression and increased N-cadherin, Vimentin and Slug expression (Fig 2C). This finding indicated that miR-140 might contribute to regulating EMT marker expression in EC in vitro. 


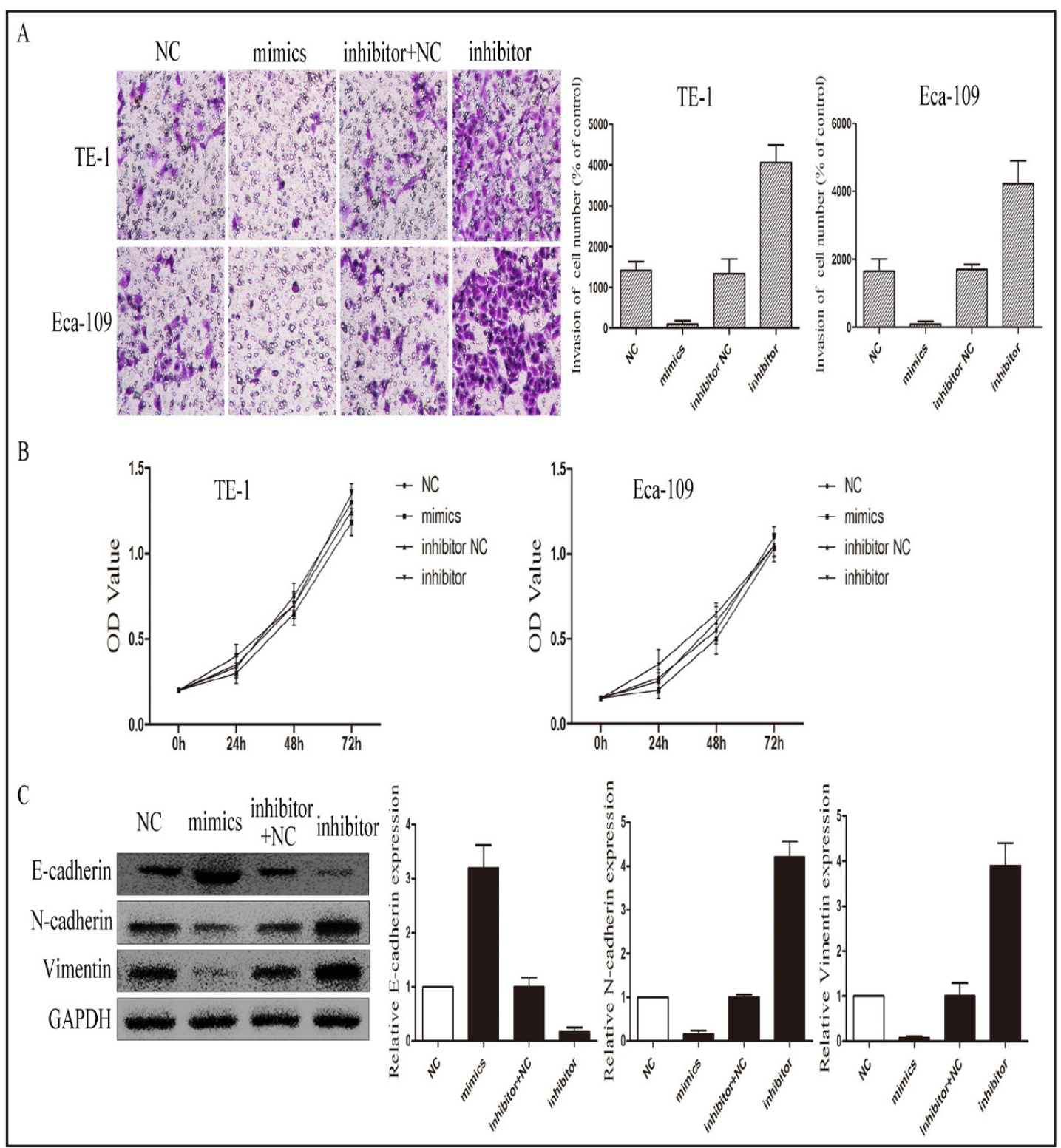

Fig. 2. miR-140 regulates EMT and EC cells invasion but has no effect on cell proliferation. A: Transwell assay was performed as described in Materials and Methods. Cells were treated with miR-140 mimics, miR140 inhibitor, NC and inhibitor NC for $24 \mathrm{~h}$. The representative images of invasive cells at the bottom of the membrane stained with crystal violet were visualized as shown. The quantifications of cell invasion were presented as percentage of cell numbers. B: The cell-proliferation assay test showed distinct differences on proliferation after manipulation of miR-140 in both TE-1 and Eca-109 cells at 12-hour, 24-hour, and 48-hour time points. C: The protein expression levels of E-cadherin, N-cadherin and Vimentin in Eca-109 cells transfected with miR-140 mimics, miR-140 inhibitor, NC and inhibitor NC were analyzed by Western-blotting. GAPDH was used as a loading control. Average values of integrated optical density (IOD) were assessed by analyzing five fields per slide and recorded in the histogram. All experiments were performed in triplicate and presented as mean \pm SEM. * indicates significant difference compared with control group $(\mathrm{P}<0.05)$. Every independent experiment was performed 3 times.

Slug is a direct target gene of miR-140

To search insights into the biological implications of miR-140 on EC tumorigenesis, we performed a bioinformatics analysis. Among hundreds of genes predicted by online miRNA 


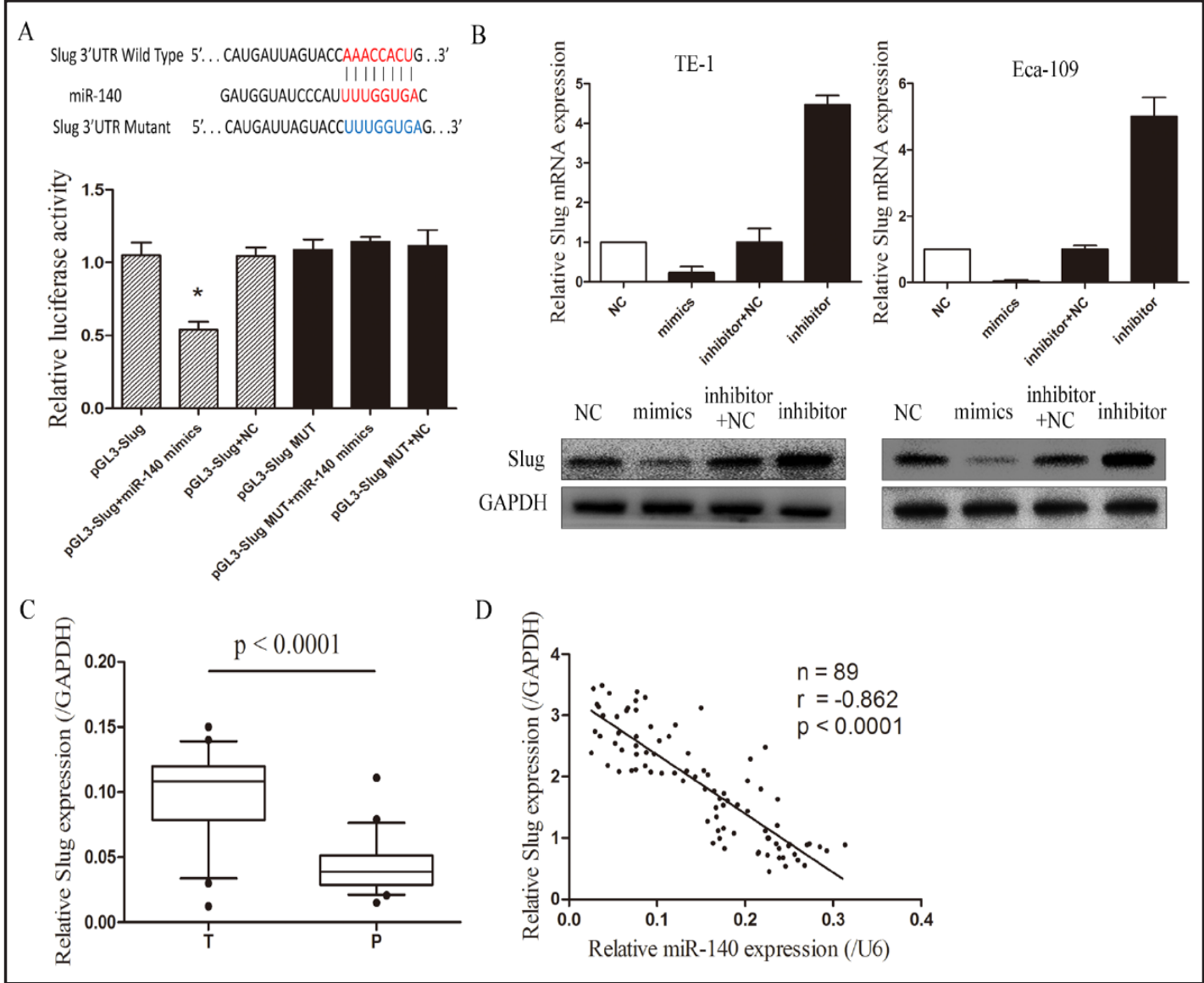

Fig. 3. miR-140 regulates Slug. A: The potential miR-140 seed region at the 3 '-UTR of Slug mRNA was computationally predicted by microRNA.org. Eca-109 cells were co-transfected with miR-140 mimics (or NC) with pGL3-Slug (or pGL3-Slug-mut) vector. Luciferase activity was normalized by the ratio of firefly and Renilla luciferase signals. B: Slug protein expression level in TE-1 and Eca-109 cells transfected with NC, miR140 mimics, inhibitor NC and miR-140 inhibitor were analyzed by using western-blotting assay. GAPDH was used as a control. All experiments were performed in triplicate and the band intensity values were analyzed by using Image J. Then, the significance in change in protein expression was analyzed by using statistical ttests. C: The mRNA levels of Slug relative to GAPDH in human EC tissues and corresponding adjacent tissues were evaluated by qRT-PCR. D: A negative correlation was found between RNA expression of miR-140 and Slug in tumor samples $(\mathrm{R}=-0.862 ; \mathrm{p}<0.0001)$. Data are represented as mean \pm SEM. * indicates $\mathrm{P}<0.05$.

target prediction algorithms, named microRNA.org (http://www.microrna.org/microrna/), miRDB (http://mirdb.org/cgi-bin/), and DIANA-MICROT (http://diana.cslab.ece.ntua.gr/ micro-CDS/), we focused on Slug, which had been reported regulating esophageal cancer progression.

To further explore the role of miR-140 on controlling Slug expression, we cloned the 3'-UTR fragment containing the predicted site into pGL3 luciferase reporter vector (pGL3Slug) according to results of prediction. The 3 '-UTR fragment with mutant sequence was also cloned as a control group (pGL3-Slug-mut) in the predicted target site. The results showed that the luciferase activity decreased in Eca-109 cells with miR-140 mimics and pGL3-Slug vectors. However, miR-140 mimics did not have any effect on luciferase activity when target cells were transfected with pGL3-Slug-mut vector (Fig. 3A). In additional, to confirm the regulatory effect of miR-140 on Slug, we performed western blot assay to detect the expression of Slug responses to the changes of miR-140 expression both in TE-1 and Eca-109 cell lines. 


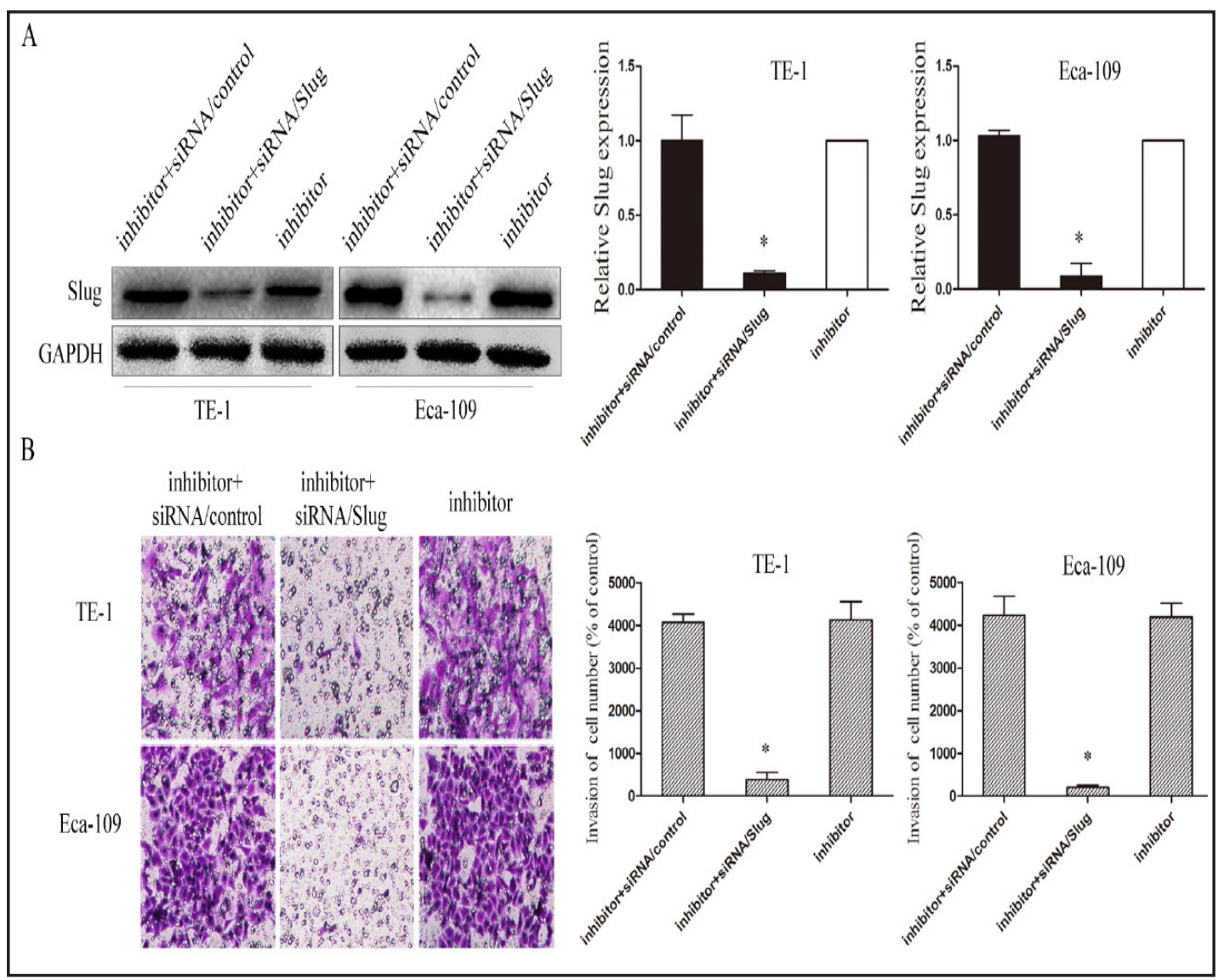

Fig. 4. Silencing of Slug reverses the anti-invasion effect of miR-140 downregulated. A: The transfection efficiency was validated by using western-blotting after both TE-1 and Eca-109 cells transfected with miR140 inhibitor and inhibitor+siRNA/Slug (the co-transfection cells of miR-140 inhibitor and siRNA/Slug) and inhibitor+siRNA/control (co-transfected with miR-140 inhibitor and siRNA/control). GAPDH was used as a control. Average values of integrated optical density (IOD) were assessed by analyzing five fields per slide and recorded in the histogram. B: Transwell assay was performed as described in Materials and Methods. Cells were treated with miR-140 inhibitor and inhibitor+siRNA/Slug (the co-transfection cells of miR-140 inhibitor and siRNA/Slug) and inhibitor+siRNA/control (co-transfected with miR-140 inhibitor and siRNA/ control) for $24 \mathrm{~h}$. The representative images of invasive cells at the bottom of the membrane stained with crystal violet were visualized as shown. The quantifications of cell invasion were presented as percentage of cell numbers. * indicates $\mathrm{P}<0.05$. Fig 1.

As shown in Fig. 3B, the assay showed a negative regulatory effect of miR-140 on Slug. Upregulated miR-140 could decrease the expression of Slug; meanwhile, down-regulated miR140 could increase Slug expression level. Here, Slug was up-regulated in esophageal cancer tissues ( $\mathrm{n}=89$ ) by using qRT-PCR (Fig. 3C). Moreover, an inverse correlation was observed between miR-140 and Slug ( $R=-0.862$; $p<0.0001$; Fig. 1D) in EC cancer samples. These results suggested that Slug gene is one of the direct targets of miR-140.

Silencing of Slug by siRNA partially reverses the effect of miR-140 downregulated

To further test whether the functional effect of miR-140 on EC cell lines was exerted via Slug, we performed a rescue experiment. As shown above, Slug was increased in cells which were transfected with miR-140 inhibitor. Thus, we silenced the expression of Slug by RNA interference. Both TE-1 and Eca-109 cells were co-transfected with miR-140 inhibitor and siRNA/Slug. The transfection cells of miR-140 inhibitor and the co-transfection cells of miR-140 inhibitor and siRNA/control were serve as the control groups. The transfection 
efficiency was confirmed by using western blot assay (Fig. 4A). Compared with the control groups, the expression level of Slug was obviously reduced both in cells co-transfected with miR-140 inhibitor and siRNA/Slug. Then, the transwell invasion assay as shown in Fig. 4B also showed that the invasive ability indeed reversed to some extent when silenced Slug in cells transfected with miR-140 inhibitor compared to control. Taken together, these results suggested that the functional effect of miR-140 on EC cell lines was dependent on Slug.

\section{Discussion}

Esophageal cancer is one of the common causes of cancer-related death in the world. The major causes of death from cancer are complications arising from metastasis. microRNAs (miRNAs) have been reported to have critical regulatory roles in cancer biology [16-18]. Specifically for EC, it is already reported that miRNAs contributes to proliferation and invasion $[19,20]$. MiR-16 induced the suppression of cell apoptosis while promote proliferation in esophageal squamous cell carcinoma [21]. miR-625 down-regulation promotes proliferation and invasion in esophageal cancer by targeting Sox2 [22]. Here, we sought to provide evidence for downexpression of miR-140 targeting of Slug to promote the invasion in EC.

miR-140 expression is decreased in EC, but the underlying mechanism in EC was unexplored. Emerging studies reported that monocyte to macrophage differentiationassociated (MMD) targeted by miR-140-5p regulates tumor growth in non-small cell lung cancer [23]. miR-140-5p suppresses BMP2-mediated osteogenesis in undifferentiated human mesenchymal stem cells [24]. Meanwhile, Slug expression is increased in EC. Slug (Snail2) is one of transcription factors that play a central role in the activation of EMT. And epithelial-to-mesenchymal transition (EMT) is considerate to be involved in migration, tumor invasion and dissemination [25-27]. Decreased E-cadherin and elevated Vimentin and $\mathrm{N}$-cadherin expression is one hallmark of EMT which is a key element in the cancer invasion [28]. Many studies established functional associations between noncoding microRNAs and key effectors of EMT occurring in the context of carcinogenesis and embryonic development, such as miR-200 [29] and miR-10b [30]. In this study, expression of miR-140 in EC specimens was significantly lower than in corresponding adjacent tissues. Up-regulated miR-140 expression resulted in increased E-cadherin expression and decreased $\mathrm{N}$-cadherin and Vimentin expression. Meanwhile, suppressed miR-140 expression resulted in decreased E-cadherin expression and increased N-cadherin and Vimentin expression.

In summary, we identified the inverse expression levels of miR-140 and Slug in our 89 EC cases, both of which were related to tumor stage and metastasis. We also provided evidence to prove the regulatory function of miR-140 on Slug by direct binding of its 3 UTR. As the limit on the number of EC samples and cell types, more elaborate studies will be necessary for further exploration of the potential role of miR-140 in development of EC. In future, miR-140-Slug pathway that we studied might be exploited in a therapeutic approach for the treatment of EC.

\section{Abbreviations}

EC (esophageal cancer); 3'-UTR (3'-untranslated regions); qRT-PCR (Quantitative Real Time- Polymerase Chain Reaction); FBS (fetal calf serum); EDTA (ethylene Diamine Tetraacetic Acid); EMT (epithelial-to-mesenchymal transition).

\section{Acknowledgments}

This research was partly supported by Zhe Lin for language revision. 
Li et al.: miR-140 in EC

\section{References}

1 Shigaki H, Baba Y, Watanabe M, Murata A, Ishimoto T, Iwatsuki M, Iwagami S, Nosho K, Baba H: Pik3ca mutation is associated with a favorable prognosis among patients with curatively resected esophageal squamous cell carcinoma. Clin Cancer Res 2013;19:2451-2459.

2 Zhang F, Yang Z, Cao M, Xu Y, Li J, Chen X, Gao Z, Xin J, Zhou S, Zhou Z, Yang Y, Sheng W, Zeng Y: Mir-203 suppresses tumor growth and invasion and down-regulates mir-21 expression through repressing ran in esophageal cancer. Cancer Lett 2014;342:121-129.

-3 Demeester SR: Epidemiology and biology of esophageal cancer. Gastrointest Cancer Res 2009;3:S2-5.

-4 Koshy M, Esiashvilli N, Landry JC, Thomas CR, Jr., Matthews RH: Multiple management modalities in esophageal cancer: Combined modality management approaches. Oncologist 2004;9:147-159.

-5 Shao Y, Zhang SQ, Quan F, Zhang PF, Wu SL: Microrna-145 inhibits the proliferation, migration and invasion of the human tca8113 oral cancer line. Oncol Lett 2013;6:1636-1640.

6 Yang L, Wang YL, Liu S, Zhang PP, Chen Z, Liu M, Tang H: Mir-181b promotes cell proliferation and reduces apoptosis by repressing the expression of adenylyl cyclase 9 (ac9) in cervical cancer cells. FEBS Lett 2014;588:124-130.

7 Billeter AT, Barnett RE, Druen D, Polk HC Jr, van Berkel VH: Microrna as a new factor in lung and esophageal cancer. Semin Thorac Cardiovasc Surg 2012;24:155-165.

-8 Yuan Y, Shen Y, Xue L, Fan H: Mir-140 suppresses tumor growth and metastasis of non-small cell lung cancer by targeting insulin-like growth factor 1 receptor. PloS One 2013;8:e73604.

-9 Song B, Wang Y, Xi Y, Kudo K, Bruheim S, Botchkina GI, Gavin E, Wan Y, Formentini A, Kornmann M, Fodstad O, Ju J: Mechanism of chemoresistance mediated by mir-140 in human osteosarcoma and colon cancer cells. Oncogene 2009;28:4065-4074.

10 Polyak K, Weinberg RA: Transitions between epithelial and mesenchymal states: Acquisition of malignant and stem cell traits. Nat Rev Cancer 2009;9:265-273.

-11 Wang Y, Wen M, Kwon Y, Xu Y, Liu Y, Zhang P, He X, Wang Q, Huang Y, Jen KY, LaBarge MA, You L, Kogan SC, Gray JW, Mao JH, Wei G: Cul4a induces epithelial-mesenchymal transition and promotes cancer metastasis by regulating zeb1 expression. Cancer Res 2014;74:520-531.

12 Liu J, Ruan B, You N, Huang Q, Liu W, Dang Z, Xu W, Zhou T, Ji R, Cao Y, Li X, Wang D, Tao K, Dou K: Downregulation of mir-200a induces emt phenotypes and csc-like signatures through targeting the betacatenin pathway in hepatic oval cells. PloS One 2013;8:e79409.

13 Thompson EW, Williams ED: Emt and met in carcinoma--clinical observations, regulatory pathways and new models. Clin Exp Metastasis 2008;25:591-592.

14 Gregory PA, Bert AG, Paterson EL, Barry SC, Tsykin A, Farshid G, Vadas MA, Khew-Goodall Y, Goodall GJ: The mir-200 family and mir-205 regulate epithelial to mesenchymal transition by targeting zeb1 and sip1. Nat Cell Biol 2008;10:593-601.

15 Oishi N, Kumar MR, Roessler S, Ji J, Forgues M, Budhu A, Zhao X, Andersen JB, Ye QH, Jia HL, Qin LX, Yamashita T, Woo HG, Kim YJ, Kaneko S, Tang ZY, Thorgeirsson SS, Wang XW: Transcriptomic profiling reveals hepatic stem-like gene signatures and interplay of mir-200c and epithelial-mesenchymal transition in intrahepatic cholangiocarcinoma. Hepatology 2012;56:1792-1803.

16 Shi L, Zhang S, Wu H, Zhang L, Dai X, Hu J, Xue J, Liu T, Liang Y, Wu G: Mir-200c increases the radiosensitivity of non-small-cell lung cancer cell line a549 by targeting vegf-vegfr2 pathway. PloS One 2013;8:e78344.

17 Lewis H, Lance R, Troyer D, Beydoun H, Hadley M, Orians J, Benzine T, Madric K, Semmes OJ, Drake R, Esquela-Kerscher A: Mir-888 is an expressed prostatic secretions-derived microrna that promotes prostate cell growth and migration. Cell Cycle 2014;13:227-239.

18 Kim J, Wu L, Zhao JC, Jin HJ, Yu J: Tmprss2-erg gene fusions induce prostate tumorigenesis by modulating microrna mir-200c. Oncogene Doi: 10.1038/onc.2013.461.

19 Akanuma N, Hoshino I, Akutsu Y, Murakami K, Isozaki Y, Maruyama T, Yusup G, Qin W, Toyozumi T, Takahashi M, Suito H, Hu X, Sekino N, Matsubara H: Microrna-133a regulates the mrnas of two invadopodia-related proteins, fscn1 and mmp14, in esophageal cancer. Brit J Cancer 2014;110:189-198.

20 Yu X, Li H, Jiang X, Guo L, Jiang W, Lu SH: Mir-203 inhibits the proliferation and self-renewal of esophageal cancer stem-like cells by suppressing stem renewal factor bmi-1. Stem Cells Dev 2014;23:576-585.

-21 Zhu Y, Xia Y, Niu H, Chen Y: Mir-16 induced the suppression of cell apoptosis while promote proliferation in esophageal squamous cell carcinoma. Cell Physiol Biochem 2014;33:1340-1348. 
22 Wang Z, Qiao Q, Chen M, Li X, Liu C, Xie Z: Mir-625 down-regulation promotes proliferation and invasion in esophageal cancer by targeting sox2. FEBS Lett 2014;588:915-921.

-23 Li W, He F: Monocyte to macrophage differentiation-associated (mmd) targeted by mir-140-5p regulates tumor growth in non-small cell lung cancer. Biochem Bioph Res Co 2014;450:844-850.

-24 Hwang S, Park SK, Lee HY, Kim SW, Lee JS, Choi EK, You D, Kim CS, Suh N: Mir-140-5p suppresses bmp2mediated osteogenesis in undifferentiated human mesenchymal stem cells. FEBS Lett 2014;588:29572963.

-25 Yuan XW, Wang DM, Hu Y, Tang YN, Shi WW, Guo XJ, Song JG: Hepatocyte nuclear factor 6 suppresses the migration and invasive growth of lung cancer cells through p53 and the inhibition of epithelialmesenchymal transition. J Bio Chem 2013;288:31206-31216.

26 Ye J, Wu D, Shen J, Wu P, Ni C, Chen J, Zhao J, Zhang T, Wang X, Huang J: Enrichment of colorectal cancer stem cells through epithelial-mesenchymal transition via cdh1 knockdown. Mol Med Rep 2012;6:507-512.

-27 Shi L, Jackstadt R, Siemens H, Li H, Kirchner T, Hermeking H: P53-induced mir-15a/16-1 and ap4 form a double-negative feedback loop to regulate epithelial-mesenchymal transition and metastasis in colorectal cancer. Cancer Res 2014;74:532-542.

28 Kitamura K, Seike M, Okano T, Matsuda K, Miyanaga A, Mizutani H, Noro R, Minegishi Y, Kubota K, Gemma A: Mir-134/487b/655 cluster regulates tgf-beta-induced epithelial-mesenchymal transition and drug resistance to gefitinib by targeting magi2 in lung adenocarcinoma cells. Mol Cancer Ther 2014;13:444-453.

-29 Paterson EL, Kazenwadel J, Bert AG, Khew-Goodall Y, Ruszkiewicz A, Goodall GJ: Down-regulation of the mirna-200 family at the invasive front of colorectal cancers with degraded basement membrane indicates emt is involved in cancer progression. Neoplasia 2013;15:180-191.

30 Ouyang H, Gore J, Deitz S, Korc M: Microrna-10b enhances pancreatic cancer cell invasion by suppressing tip30 expression and promoting egf and tgf-beta actions. Oncogene 2014;33:4664-4674. 